\title{
NEW ROLE OF TLR4 AS A MEDIATOR FOR INFLAMMATION-APOPTOSIS SIGNALING
} PATHWAY IN NECROTIZING ENTEROCOLITIS

\author{
B. Liu ${ }^{1}$, P.Q. Bao ${ }^{1}$, B. Zhou ${ }^{2}$, K.L. Chen ${ }^{2}$, R. Wang ${ }^{2}$, T.Z. Hu ${ }^{1}$, Y. Li ${ }^{1}$ \\ ${ }^{I}$ Pediatric Surgery, ${ }^{2}$ Lab of Digestive Surgery, West China Hospital, Sichuan University, Chengdu, China
}

Background and aims: Inflammatory key receptor TLR4 has been proved to be critical in the pathogenesis of necrotizing enterocolitis (NEC).Latest study revealed TLR4 also mediate apoptotic pathways. This study was to investigate the new role of TLR4 as a mediator for inflammation-apoptosis signaling pathways in NEC.

Methods: TLR4 deficient (C57BL/10ScnJ) and wild-type (C57BL/10J) mice were used. NEC was induced by a combination of formula gavage and cold hypoxia stress with LPS. Intestinal inflammation was evaluated by HE staining; The expression of inflammtory genes including TLR4, ReLA/p65, TNF- $\alpha$, IL-6, 10, IL-1ra and apoptotic genes of Caspase 8, 9, 3 were quantitatively determined by realtime RT-PCR. Intestinal apoptosis was examined by TUNEL.

Results: Pathological study showed inflammation was significantly reduced in TLR4 deficient mice; Realtime RT-PCR showed in TLR4-Def-NEC mice, ReLA/p65 and Caspase 8 were down-regulated, Caspase 9 was up-regulated but Caspase 3 remained unchanged; TNF- $\alpha$ could not be detected firstly but was significantly up-regulated after the inductoin of NEC. In TLR4-WT-NEC group, TLR4, ReLA/p65 and Caspase 8 were both up-regulated while TNF- $\alpha$ and Caspase 9 were down-regulated; Compared with TLR4 WT-NEC group, ReLA/p65,TNF- $\alpha$ and caspase 8 were down-regulated while Caspase 9 significantly upregulated in TLR4 def-NEC mice. TUNEL showed the number of apoptotic cells was less in NEC group in both mice strains; TLR4-Def NEC mice have higher numbers of apoptotic cells than that in TLR4-WT NEC mice.

Conclusions: TLR4-mediated inflammation-apoptotic signaling pathways affect the development of NEC, TLR4 mediate apoptosis by activating Caspase 8 rather than Caspase 9. 